\title{
Pre-engraftment clinically significant CMV infection after allogeneic hematopoietic stem cell transplantation and its impact on engraftment
}

Marcia Garnica ( $\sim$ marciagarnica@hucff.ufrj.br)

Universidade Federal do Rio de Janeiro https://orcid.org/0000-0003-1067-6680

\section{Sylvia Dalcolmo}

Universidade Federal do Rio de Janeiro

\section{Bianca Gaio}

Universidade Federal do Rio de Janeiro

\section{Andreia Almeida}

DASA - Complexo Hospitalar de Niterói

Juliana Rivello

DASA - Complexo Hospitalar de Niterói

Ricardo Bigni

DASA - Complexo Hospitalar de Niterói

\section{Marcia Rejane Valentim}

DASA - Complexo Hospitalar de Niterói

Maria Claudia Moreira

Instituto Nacional de Câncer https://orcid.org/0000-0002-8376-7199

Angelo Maiolino

Universidade Federal do Rio de Janeiro https://orcid.org/0000-0003-0140-9548

\section{Article}

Keywords: Stem cell transplantation, cytomegalovirus, viremia, antiviral therapy, engraftment

Posted Date: June 30th, 2021

DOl: https://doi.org/10.21203/rs.3.rs-573650/v1

License: (c) (i) This work is licensed under a Creative Commons Attribution 4.0 International License.

Read Full License 


\section{Abstract}

Pre-engraftment cytomegalovirus infection $(\mathrm{CMVi})$ is a challenge in patients receiving allogeneic stem cell transplantation (Allo-HCT), as few data have been reported on its clinical importance. This study describes the clinical outcomes of pre-engraftment CMVi and compares them with those of episodes developing after engraftment in HCT patients.

We performed a retrospective study of patients who underwent Allo-HCT from 2016 to 2020, including 111 recipients monitored by real-time PCR assay. Clinically significant CMVi (csCMVi) was documented in 81 (73\%) patients. There were $29(26 \%)$ cases of pre-engraftment csCMVi. No significant difference was observed regarding virological features, but patients with pre-engraftment csCMVi had a delayed start in treatment $(24$ vs. 12 days, $p<0.001)$ compared with those with postengraftment events. Preengraftment csCMVi was associated with a delay in engraftment (20 vs. 16 days, $p=0.02$ ) and worse overall survival ( $54 \%$ vs. $73 \%$ 1-year $O S ; p=0.020$ ) than postengraftment events. In conclusion, preengraftment csCMVi occurred in $26 \%$ of our patients and was associated with engraftment delay and worse overall survival. Close monitoring of CMV DNAemia is necessary to identify these patients earlier. Prospective studies, including patients with letermovir prophylaxis, are necessary to define the standard in the management and care of this population.

\section{Introduction}

Cytomegalovirus infection (CMVi) leads to high morbidity in patients undergoing allogeneic stem cell transplantation (Allo-HCT) and impacts clinical outcomes, including overall survival. $(1,2,3) \mathrm{CMVi}$ occurs in 70 to $80 \%$ of Allo-HCT patients after HCT. (4) Most CMV reactivations occur in the first 100 days after HSCT at a median of 50 days post HCT, but some patients present very early CMV reactivation, sometimes before engraftment. $(1,5)$

Approximately $25 \%$ of documented CMV reactivations have spontaneous clearance (i.e., without the need for therapy). (6) Nevertheless, in others, viral replication gives rise to clinically significant CMV infection (csCMVi), and a pre-emptive approach with antiviral therapy is needed to prevent CMV organ disease (710)

csCMVi documented pre-engraftment is challenging to treat. Anti-CMV drugs (usually intravenous ganciclovir or oral valganciclovir) have significant adverse effects, including myelotoxicity. (11-13) Foscarnet has severe side effects, especially nephrotoxicity, and in some countries, its access is difficult. (8)

In this study, we describe the frequency pf and risk factors for pre-engraftment csCMVi in HCT patients and evaluate its impact on the time of engraftment and overall survival.

\section{Patients And Methods}


Consecutive adult patients who underwent Allo-HCT at Complexo Hospitalar de Niteroi between 2016 and 2020 were included in the observational retrospective cohort. CMV-seronegative recipients allografted with $\mathrm{CMV}$-seronegative donors, those receiving anti-CMV prophylaxis, or patients who died before $\mathrm{D}+30$ after transplant were excluded. CMV viral loads (time after HCT to reactivation, initial viral load, highest viral load within the event, duration of viremia, and viremia clearance) were obtained for the first episode of following HCT for each patient. For this study, outcomes were analyzed until $D+100$. The local Institutional Review Board approved the study (CEP Hospital 9 de Juho, CAAE number 54941216.0.3001.5455), and the study was conducted according to guidelines of the Declaration of Helsinki.

Patients underwent weekly surveillance for CMV viremia by a CMV real-time polymerase chain reaction (PCR) kit (produced by Qiagen - CMV amplification Reagent kit - Abbott) in plasma. (14) This commercial test provides a limit of detection of $31 \mathrm{IU} / \mathrm{mL}(95 \% \mathrm{Cl})$. In this study, DNAemia was reported in quantitative international units (IU) per milliliter. The screening started in the first week after SCT and was repeated weekly until $D+100$ and after $D+100$ if immunosuppression was maintained.

Preemptive therapy was initiated at the treating physician's discretion, and it was based upon positive surveillance tests and the patient's risk group. Suggested thresholds were CMV viremia of more than 150 $\mathrm{IU} / \mathrm{mL}$ for high-risk (cord- blood, haploidentical, HLA-mismatched or T-cell depleted Allo-HCT or receiving $>1 \mathrm{mg} / \mathrm{kg}$ corticosteroids) patients and more than $500 \mathrm{lU} / \mathrm{mL}$ for low-risk patients (all HCT that do not meet high risk criteria). (10) (Val)gancyclovir is standard therapy unless the episode occurs before engraftment or during neutropenia. Foscarnet is the drug of choice if ganciclovir is contraindicated. (8)

All the patients received antiviral prophylaxis with acyclovir, and none of them received anti-CMV prophylaxis. Quinolone prophylaxis is not routinely used. Antifungal prophylaxis is recommended in highrisk patients (acute leukemia, previous invasive fungal infection, or long estimated neutropenia). Cefepime is the frontline empiric treatment for febrile neutropenia unless there is a multiresistant pathogen history (colonization or previous infection) or a clinically complicated presentation. In these situations, empirical therapy is adapted by risk and de-escalated when possible and at the discretion of the treating physician.

Data collection

CMV episodes were described considering the time after SCT to reactivation, initial viral load, highest viral load within the event, duration of viremia, treatment, and viremia clearance after the start of anti-CMV therapy.

We collected HCT characteristics (baseline disease and its status, preconditioning absolute lymphocyte count), donor type and stem cell source, conditioning, and graft versus host disease (GVHD) prophylaxis.

In this analysis, patients with csCMVi detected pre-engraftment were compared with those with csCMVi detected post engraftment. Engraftment was defined as absolute neutrophil count $>500 \mathrm{cels} / \mathrm{mm}^{3}$ on 
three consecutive days.

For outcome analysis, we collected data regarding CMV episode outcomes (CMV clearance) and HCT outcomes: time to engraftment and overall mortality at D100 and 1 year post HCT.

\section{Definitions}

CMV episode was defined as detecting CMV DNA at any level in one or more plasma samples. Clinically significant CMV infection (csCMVi) was defined as CMV disease or CMV viremia leading to preemptive treatment. (9) CMV clearance was defined as the documentation of 2 negative PCR values. The overall duration of CMV viremia was between the first detection and the first negative (indetectable) PCR result. Viremia clearance was defined as the interval from the date of the initiation of anti-CMV therapy to clearance. (6)

aGVHD was diagnosed and graded as reported by the modified Glucksberd criteria. (15) All-cause mortality was calculated at $D+100$ and 1 year post $\mathrm{HCT}$.

Statistical analyses

Categorical and continuous numeric variables were expressed as frequencies and medians and explored using the chi-square or Fisher exact test, Wilcoxon rank-sum test, or Spearman correlation test. Time to event was estimated by Kaplan-Meier analysis and analyzed by the log-rank test. Risk factors for preengraftment CsCMVi were assessed by logistic regression analysis. Cox proportional hazard regression was used to assess risk factors for time to engraftment and overall survival. A p-value lower than $5 \%$ was considered significant. All analyses were performed with the IBM SPSS statistical package (SPSS Statistics for MAC, Version 27, by IBM Corporation).

\section{Results}

During the study, 111 stem cell transplant patients were enrolled. The cohort's median age was 40 years (ranging from 18 to 72 years old). CMV seropositivity was found in $95 \%$ of recipients. Acute myeloid leukemia and acute lymphoid leukemia were the most frequent underlying diseases $(n=45,41 \%$ and $n=$ $22,20 \%$, respectively). The numbers of haploidentical, unrelated and fully matched related donors were $45(40 \%), 33(30 \%)$, and $33(30 \%)$, respectively. The most frequent GVHD prophylactic therapy was cyclosporine-based therapy $(n=72 ; 65 \%)$. The time to engraftment from infusion was $D+17(+2-+41)$, the median follow-up was 235 days $(33-1,604)$, and $78 \%$ of our patients had a follow-up longer than 100 days (Table 1 ). 
Table 1

Characteristics of the study patients

\section{Variables}

Patient sex, male
Patient age, median (range)

Underlying disease

Acute myeloid leukemia

Acute lymphoblastic leukemia

Lymphomas

Myelofibrosis

Myelodysplastic syndromes

Aplastic anemia

Chronic myeloid leukemia

Others*

Disease status at HSCT

1st Complete remission

2nd Complete remission

Relapse/progression

Active disease ${ }^{\star \star}$

Recipient/donor CMV serology

$\mathrm{R}+\mathrm{D}+$

$\mathrm{R}+\mathrm{D}-$

$\mathrm{R}-\mathrm{D}+$

Absolute lymphocytes count preconditioning

$<1000$ lymphocytes
All Patients

$N=111$

$62(55.9)$

$40(18-72)$

45 (40.5)

$22(19.8)$

15 (13.4)

$10(9)$

7 (6.3)

5 (4.5)

4 (3.6)

$3(2.7)$

37 (33.3)

$29(26.1)$

20 (18)

$24(21.6)$

$96(86.5)$

$10(9)$

$5(4.5)$

$1436(240-32,159)$

$28(26)$

Unless otherwise noted, all data are $\mathrm{n}(\%)$

aGVHD, acute graft-versus host disease; ATG, anti-thymocyte globulin; CyA, cyclosporine; D, donor; $\mathrm{HCT}$, hematopoietic stem cell transplant; $R$, recipient; PBSC, peripheral blood stem cell

*ATLL $n=2 ; S C D=1 ;$ ** Active disease: aplastic anemia, MDS, myelofibrosis 


\begin{tabular}{|c|c|}
\hline Variables & $\begin{array}{l}\text { All Patients } \\
\mathrm{N}=111\end{array}$ \\
\hline \multicolumn{2}{|l|}{ Conditioning regimen, $\mathrm{n}(\%)$} \\
\hline Myeloablative & $74(67)$ \\
\hline \multicolumn{2}{|l|}{ T cell depletion, $\mathrm{n}(\%)$} \\
\hline ATG & $48(43.2)$ \\
\hline \multicolumn{2}{|l|}{ Stem cell source } \\
\hline Bone marrow & $49(44.1)$ \\
\hline PBSC & $62(55.9)$ \\
\hline \multicolumn{2}{|l|}{ Type of donor } \\
\hline HLA-matched related donor & $33(29.7)$ \\
\hline HLA-mismatched related donor (haploidentical) & $45(40.5)$ \\
\hline HLA-matched unrelated donor & $33(29.7)$ \\
\hline \multicolumn{2}{|c|}{ Cellularity of the donor progenitor infusion (per $10^{6}$ cells $/ \mathrm{kg}$ ) } \\
\hline CD34 & $4.49(0.29-19.8)$ \\
\hline Mononuclear & $4.58(1.08-24)$ \\
\hline \multicolumn{2}{|l|}{ aGVHD prophylaxis } \\
\hline CyA-based regimen & $72(64.9)$ \\
\hline Tacrolimus-based regimen & $39(35.1)$ \\
\hline Mycophenolate mofetil use & $50(45)$ \\
\hline \multicolumn{2}{|l|}{ aGVHD } \\
\hline Grade $0-1$ & $64(57.7)$ \\
\hline Grade II-IV & $47(42.3)$ \\
\hline Follow-up, post-HCT, median (days) & $235(33-1604)$ \\
\hline \multicolumn{2}{|l|}{ Unless otherwise noted, all data are $\mathrm{n}(\%)$} \\
\hline \multicolumn{2}{|c|}{$\begin{array}{l}\text { aGVHD, acute graft-versus host disease; ATG, anti-thymocyte globulin; CyA, cyclosporine; D, donor; } \\
\text { HCT, hematopoietic stem cell transplant; R, recipient; PBSC, peripheral blood stem cell }\end{array}$} \\
\hline${ }^{*} A T L L n=2 ; S C D=1 ; * \star$ Active disease: aplastic & \\
\hline
\end{tabular}

csCMVi was documented in 81 (73\%) patients. CMV disease (documented by biopsy) occurred in 7 patients ( 5 gastrointestinal CMVi, 1 pneumonia and 1 documented CMVi in the bladder). 
Pre-engraftment csCMVi was seen in 29 patients (3 preinfusion and 26 pre-engraftment), and 52 patients had csCMVi after engraftment (53\%). In 20 (27\%) patients, there was no documentation of CMV during follow-up.

Therapy for pre-engraftment csCMVi was mainly started after engraftment $(79 \%)$. The drug was initiated before engraftment in only three patients, all of whom received foscarnet. In the other 3 , ganciclovir was started concomitantly with engraftment. The treatment of pre-engraftment csCMVi was started a median of 24 days after CMV DNAemia was first documented. In patients with postengraftment csCMVi, the median time to start therapy was 12 days $(p<0.001)$. The CMV viral load at the start of therapy was similar in pre- and postengraftment events (2184 vs. $1742 \mathrm{IU} / \mathrm{ml} ; \mathrm{p}=0.637$ ).

The patients' clinical characteristics and virological features of csCMVi are detailed in Table 2. There were significant differences in the source of stem cells, CD34 cellularity of donor progenitors, time to engraftment, and overall mortality between pre-engraftment and postengraftment csCMVi patients. Regarding virological features, no significant difference was observed. 
Table 2

Clinical and virological features and outcomes of pre-engraftment and postengraftment csCMVi

\section{Variables}

Patient sex, male

Patient age, median (range),

Underling disease

Myeloid vs. lymphoid hematological disease

BD status at HSCT

1st or 2nd Complete remission

Relapse/progression

*Active disease

Donor/recipient CMV serology

$\mathrm{R}+\mathrm{D}+$

$\mathrm{R}+\mathrm{D}-$

$\mathrm{R}-\mathrm{D}+$

Absolute lymphocyte count preconditioning

Stem cell source

Bone marrow

PBSC

Type of donor

HLA-matched related donor

HLA-mismatched related (haploidentical)

HLA-unrelated donor

Conditioning regimen

Myeloablative

Unless otherwise noted, all data are $\mathrm{n}(\%)$

aGVHD, acute graft-versus host disease; ATG, anti-thymocyte globulin; CyA, cyclosporine; csCMVi, clinically significant cytomegalovirus infection; D, donor; HCT, hematopoietic stem cell transplant; R, recipient; PBSC, peripheral blood stem cell

\section{Preengraftment} csCMVi

\section{$\mathrm{N}=29$}

$15(51.7)$

17 vs. 10

$39(18-70)$

33 vs. 17

0.807

0.388

22 (75.9)

3 (10.3)

4 (13.8)

12 (23)

31 (59.6)

8 (15.4)

26 (89.7)

44 (84.6)

2 (6.9)

7 (13.5)

1 (3.4)

1 (1.9)

1,216 (389$122,944)$

1,452 (240$16,236)$

0.621

18 (62)

15 (28.8)

11 (37.9)

36 (69.2)

0.283

$5(17.2)$

15 (28.8)

13 (44.8)

25 (48)

11 (37.9)

12 (23)

$20(68.9)$

32 (61.5)

0.63 


\begin{tabular}{|c|c|c|c|}
\hline Variables & $\begin{array}{l}\text { Preengraftment } \\
\text { csCMVi } \\
\mathrm{N}=29\end{array}$ & $\begin{array}{l}\text { Postengraftment } \\
\mathrm{N}=52\end{array}$ & $\begin{array}{l}\mathrm{P} \\
\text { value }\end{array}$ \\
\hline \multicolumn{4}{|l|}{ T cell depletion, $\mathrm{n}(\%)$ : } \\
\hline ATG & $14(48.3)$ & $20(38.5)$ & 0.472 \\
\hline \multicolumn{4}{|l|}{$\begin{array}{l}\text { Cellularity of the donor progenitor infusion (per } \\
10^{6} \mathrm{cells} / \mathrm{kg} \text { ) }\end{array}$} \\
\hline CD34 & $3.23(0.29-10.21)$ & $\begin{array}{l}3,37(0.41- \\
19.81)\end{array}$ & 0.033 \\
\hline Mononuclear & $3.78(1.25-14.58)$ & $\begin{array}{l}5.26(1.08- \\
23.97)\end{array}$ & 0.115 \\
\hline \multicolumn{4}{|l|}{ aGVHD prophylaxis } \\
\hline CyA-based regimen & $21(72.4)$ & $31(59.6)$ & 0.329 \\
\hline Tacrolimus-based regimen & $8(27.6)$ & $21(40.4)$ & \\
\hline Mycophenolate mofetil use & $14(48.3)$ & $25(48)$ & 0.816 \\
\hline Initial CMV DNA load in IU/mL & $103(31-717)$ & $103(31-622)$ & 0.822 \\
\hline CMV DNA peak load in IU/mL & $\begin{array}{l}5,447 \text { (158- } \\
234,161 \mathrm{I})\end{array}$ & $\begin{array}{l}4,392(195- \\
379,669)\end{array}$ & 0.911 \\
\hline Duration of CMV DNAemia in days & $51(14-254)$ & $49(11-235)$ & 0.388 \\
\hline Days from initial CMV DNA and therapy, median & $24(2-60)$ & $12(0-46)$ & $\begin{array}{l}< \\
0.001\end{array}$ \\
\hline Viremia clearance, days (median) & $24(7-240)$ & $33(6-215)$ & 0.374 \\
\hline CMV disease & $3(10)$ & $4(7.7)$ & \\
\hline $\mathrm{GI}$ & 2 & 3 & \\
\hline Bladder & 0 & 1 & \\
\hline Lung & 1 & 0 & \\
\hline Day of engraftment & $20(12-41)$ & $16(10-33)$ & $\begin{array}{l}< \\
0.001\end{array}$ \\
\hline Engraftment failure & $1(3.4)$ & $2(3.8)$ & \\
\hline
\end{tabular}

Unless otherwise noted, all data are $\mathrm{n}(\%)$

aGVHD, acute graft-versus host disease; ATG, anti-thymocyte globulin; CyA, cyclosporine; csCMVi, clinically significant cytomegalovirus infection; D, donor; HCT, hematopoietic stem cell transplant; R, recipient; PBSC, peripheral blood stem cell 


\begin{tabular}{|c|c|c|c|}
\hline Variables & $\begin{array}{l}\text { Preengraftment } \\
\text { csCMVi } \\
\mathrm{N}=29\end{array}$ & $\begin{array}{l}\text { Postengraftment } \\
\mathrm{N}=52\end{array}$ & $\begin{array}{l}P \\
\text { value }\end{array}$ \\
\hline aGVHD & & & 0.156 \\
\hline Grade $0-1$ & $18(62)$ & $25(48)$ & \\
\hline Grade II-IV & $10(34.5)$ & $27(51.9)$ & \\
\hline Corticosteroid therapy & $13(44.8)$ & $30(57.7)$ & 0.25 \\
\hline Overall mortality & $14(48.3)$ & $12(23)$ & 0.026 \\
\hline \multicolumn{4}{|c|}{ Unless otherwise noted, all data are $\mathrm{n}(\%)$} \\
\hline \multicolumn{4}{|c|}{$\begin{array}{l}\text { aGVHD, acute graft-versus host disease; ATG, anti-thymocyte globulin; CyA, cyclosporine; csCMVi, } \\
\text { clinically significant cytomegalovirus infection; D, donor; HCT, hematopoietic stem cell transplant; } R \text {, } \\
\text { recipient; PBSC, peripheral blood stem cell }\end{array}$} \\
\hline
\end{tabular}

Pre-engraftment csCMVi was associated with prolonged time to engraftment (median time to engraftment was 20 vs. 16 days; $p=0.02$, by log-rank) and worse overall survival (OS) than postengraftment csCMVi (Figs. 1 and 2). In a Cox regression model, including stem cell source and CD34 cellularity, pre-engraftment csCMVi was still a significant risk factor for later engraftment (Table 3).

Table 3

Time to engraftment probability by Cox model regression

\begin{tabular}{|lllll|}
\hline Variable & Unadjusted HR & P value & Adjusted HR & P value \\
\hline CD34 cellularity & 1.04 & 0.09 & 1.05 & 0.199 \\
\hline Stem cell source & 1.62 & 0.016 & 0.94 & 0.844 \\
\hline Pre-engraftment csCMVi & 2.01 & 0.005 & 2.03 & 0.012 \\
\hline csCMVi, clinically significant cytomegalovirus infection & & \\
\hline
\end{tabular}

D +100 and 1 -year OS were $71 \%$ vs. $92 \%$ and $54 \%$ vs. $73 \%$ in pre-engraftment csCMVi and postengraftment csCMVi patients, respectively ( $p=0.020$, by log-rank). In multivariate Cox regression, preengraftment csCMVi remained an independent prognostic factor $(p=0.007)$ in a model that included baseline diseases, baseline disease status, age, donor type, conditioning, stem cell source, CD34 cellularity, engraftment time, and aGVHD grade.

\section{Discussion}

In this study, we addressed the frequency and clinical impact of pre-engraftment CMVi. We noted that $26 \%$ of our patients had csCMVi before engraftment, and this event had a negative effect on time to engraftment and overall survival. In this study, we analyzed only csCMVi. In the study of CMV disease or 
CMVi that requires preemptive therapy, $\mathrm{CsCMVi}$ is the preferred primary endpoint of studies reporting CMV post HCT. (9)

When quantitative PCR was not feasible, the documentation of CMV reactivation during neutropenia was a challenge. Episodes were mostly determined through tissue biopsy. $(16,17)$ Currently, the monitoring of CMVi by quantitative PCR is routine, including during the pre-engraftment HCT phase and early CMVi, as quantitative PCR can be easily performed. $(8,16)$ Therefore, many questions about pre-engraftment CMVi need answers, including the frequency of pre-engraftment CMVi, its clinical importance, and how to treat this condition.

Few studies have previously addressed pre-engraftment CMVi, and none have reported csCMVi. $(18,19)$ Two publications addressed pre-engraftment CsCMVi in the last two years. $(18,19)$ In both, patients were closely monitored by quantitative PCR starting within the first week after HCT, but the incidence of preengraftment CMVi was in these studies was different: Solano and colleagues reported a pre-engraftment CMVi incidence of $19 \%$, whereas Martin and colleagues reported an incidence of $6.5 \%$. Our cohort had an overall csCMVi incidence (73\%) that was similar to that of Solano et al. but a higher incidence of preengraftment csCMVi (26\%), even when analyzing only csCMVi episodes. Several differences in terms of HCT population characteristics can be noted, including the frequency of CMV seropositivity in our recipients, transplant modality, and baseline disease. $(4,20)$ These differences probably contributed to the variation in pre-engraftment CMVi incidences.

Similar to Solano et al., we could not identify risk factors for pre-engraftment csCMVi by considering preHCT characteristics, the cellularity of donor progenitor infusion, conditioning strategy, or aGVHD prophylaxis. In addition, no differences in terms of viral features comparing pre- vs. postengraftment episodes were observed in Solano's cohort and our cohort. In our study, the frequency of tissue-invasive CMV disease was higher than that in other studies, but the frequencies of pre- and postengraftment csCMVi were similar.

Our series observed that pre-engraftment csCMVi was associated with a delay in engraftment compared to postengraftment csCMVi. This could not be attributed to the myelotoxicity of therapy, as only 3 of 29 pre-engraftment csCMVi patients started the treatment before engraftment. All of them were treated during neutropenia with foscarnet. Anti-CMV therapy was delayed in pre-engraftment compared with postengraftment events. Martin and colleagues also observed a delayed start of CMV treatment in patients with pre-engraftment CMVi, but no difference in engraftment was observed between those who started therapy before engraftment and those who started therapy after engraftment. It is important to highlight that Martin and colleagues did not compare pre-engraftment to postengraftment events. We hypothesize that the virus had an important role in the engraftment delay in our cohort, as the adjusted model maintained the significance of pre-engraftment csCMVi as a risk factor, and CMV is a well-known myelotoxic pathogen. $(21,22)$

Cytomegalovirus replication is a possible contributor to acute GVHD development, as demonstrated by Cantoni et al. (23) In our series, the frequency of aGVHD was not different in pre-and postengraftment 
events.

CMVi has been associated with higher nonrelapse mortality in Allo-HCT. $(1,5)$ Unlike Solano, we observed a negative impact of pre-engraftment csCMVi on overall survival, and this effect was sustained months after the csCMVi episode. Overall survival post HCT depends on several factors, but it is feasible that preengraftment csCMVi can serve as an indicator of high-risk patients.

Our study has limitations. The first is the retrospective and single-center design. As there is a lack of CMV treatment standardization and threshold viremia to indicate treatment, our results may not be extrapolated to other transplant centers.

This study described a potentially negative impact on outcome (engraftment delay and worse overall survival) in HCT patients who experienced pre-engraftment csCMVi. Close and early monitoring of CMV DNAemia after transplantation is needed to identify these patients. Prospective studies, including patients with letermovir prophylaxis, are necessary to define this population's standard in management and care. Currently, letermovir prophylaxis is the standard of care for high-risk patients $(8,24,25)$, and an early start of prophylactic therapy could be an effective intervention to reduce pre-engraftment csCMVi, especially in the context of high incidences of csCMV.

\section{Declarations}

Acknowledgments: $\mathrm{BG}$ received grants from Fundacao de Amparo a Pesquisa do Rio de Janeiro (FAPERJ)

Competing Interests statement: None of the authors has any conflicts of interest (direct or indirect) concerning the contents of the manuscript.

\section{References}

1. Green ML, Leisenring W, Xie H, Mast TC, Cui Y, Sandmaier BM, et al. Cytomegalovirus viral load and mortality after haemopoietic stem cell transplantation in the era of pre-emptive therapy: a retrospective cohort study. Lancet Haematol. 2016 Mar;3(3):e119-27. doi: 10.1016/S23523026(15)00289-6. Epub 2016 Feb 20. PMID: 26947200; PMCID: PMC4914379.

2. Webb BJ, Harrington R, Schwartz J, Kammerer J, Spalding J, Lee E, et al. The clinical and economic impact of cytomegalovirus infection in recipients of hematopoietic stem cell transplantation. Transpl Infect Dis. 2018 Oct;20(5):e12961. doi: 10.1111/tid.12961. Epub 2018 Jul 20. PMID: 29975816.

3. Fang J, Su Y, Zavras PD, Raval AD, Tang Y, Perales MA, et al. Impact of Preemptive Therapy for Cytomegalovirus on Hospitalizations and Cost after Hematopoietic Stem Cell Transplantation. Biol Blood Marrow Transplant. 2020 Oct;26(10):1937-1947. doi: 10.1016/j.bbmt.2020.06.025. Epub 2020 Jul 5. PMID: 32640313. 
4. Stern L, Withers B, Avdic S, Gottlieb D, Abendroth A, Blyth E, Slobedman B. Human Cytomegalovirus Latency and Reactivation in Allogeneic Hematopoietic Stem Cell Transplant Recipients. Front Microbiol. 2019 May 28;10:1186. doi: 10.3389/fmicb.2019.01186. PMID: 31191499; PMCID: PMC6546901.

5. Teira P, Battiwalla M, Ramanathan M, Barrett AJ, Ahn KW, Chen M, et al. Early cytomegalovirus reactivation remains associated with increased transplant-related mortality in the current era: a CIBMTR analysis. Blood. 2016 May 19;127(20):2427-38. doi: 10.1182/blood-2015-11-679639. Epub 2016 Feb 16. PMID: 26884374; PMCID: PMC4874224.

6. Camargo JF, Kimble E, Rosa R, Shimose LA, Bueno MX, Jeyakumar N, et al. Impact of Cytomegalovirus Viral Load on Probability of Spontaneous Clearance and Response to Preemptive Therapy in Allogeneic Stem Cell Transplantation Recipients. Biol Blood Marrow Transplant. 2018 Apr;24(4):806-814. doi: 10.1016/j.bbmt.2017.11.038. Epub 2017 Dec 5. PMID: 29217388.

7. Erard V, Guthrie KA, Seo S, Smith J, Huang M, Chien J, et al. Reduced Mortality of Cytomegalovirus Pneumonia After Hematopoietic Cell Transplantation Due to Antiviral Therapy and Changes in Transplantation Practices. Clin Infect Dis. 2015 Jul 1;61(1):31 - 9. doi: 10.1093/cid/civ215. Epub 2015 Mar 16. PMID: 25778751; PMCID: PMC4542910.

8. Ljungman P, de la Camara R, Robin C, Crocchiolo R, Einsele H, Hill JA, et al. 2017 European Conference on Infections in Leukaemia group. Guidelines for the management of cytomegalovirus infection in patients with haematological malignancies and after stem cell transplantation from the 2017 European Conference on Infections in Leukaemia (ECIL 7). Lancet Infect Dis. 2019 Aug;19(8):e260-e272. doi: 10.1016/S1473-3099(19)30107-0. Epub 2019 May 29. PMID: 31153807.

9. Ljungman P, Boeckh M, Hirsch HH, Josephson F, Lundgren J, Nichols G, et al. Disease Definitions Working Group of the Cytomegalovirus Drug Development Forum. Definitions of Cytomegalovirus Infection and Disease in Transplant Patients for Use in Clinical Trials. Clin Infect Dis. 2017 Jan 1;64(1):87-91. doi: 10.1093/cid/ciw668. Epub 2016 Sep 28. PMID: 27682069.

10. Einsele $H$, Ljungman P, Boeckh M. How I treat CMV reactivation after allogeneic hematopoietic stem cell transplantation. Blood. 2020 May 7;135(19):1619-1629. doi: 10.1182/blood.2019000956. PMID: 32202631 ; PMCID: PMC7484743.

11. Goodrich JM, Bowden RA, Fisher L, Keller C, Schoch G, Meyers JD. Ganciclovir prophylaxis to prevent cytomegalovirus disease after allogeneic marrow transplant. Ann Intern Med. 1993 Feb 1;118(3):1738. doi: 10.7326/0003-4819-118-3-199302010-00003. PMID: 8380242.

12. Goodrich JM, Mori M, Gleaves CA, Du Mond C, Cays M, Ebeling DF, et al. Early treatment with ganciclovir to prevent cytomegalovirus disease after allogeneic bone marrow transplantation. $\mathrm{N}$ Engl J Med. 1991 Dec 5;325(23):1601-7. doi: 10.1056/NEJM199112053252303. PMID: 1658652.

13. Bacigalupo A, van Lint MT, Tedone E, Moro F, Sanna MA, Longren M, et al.. Early treatment of CMV infections in allogeneic bone marrow transplant recipients with foscarnet or ganciclovir. Bone Marrow Transplant. 1994 Jun;13(6):753-8. PMID: 7920310. 
14. Clari MÁ, Bravo D, Costa E, Muñoz-Cobo B, Solano C, Remigia MJ, et al. Comparison of the new Abbott Real Time CMV assay and the Abbott CMV PCR Kit for the quantitation of plasma cytomegalovirus DNAemia. Diagn Microbiol Infect Dis. 2013 Feb;75(2):207-9. doi: 10.1016/j.diagmicrobio.2012.10.010. Epub 2012 Nov 20. PMID: 23182073.

15. Przepiorka D, Weisdorf D, Martin P, Klingemann HG, Beatty P, Hows J, Thomas ED. 1994 Consensus Conference on Acute GVHD Grading. Bone Marrow Transplant. 1995 Jun;15(6):825-8. PMID: 7581076.

16. Granot N, Storb R. History of hematopoietic cell transplantation: challenges and progress. Haematologica. 2020 Dec 1;105(12):2716-2729. doi: 10.3324/haematol.2019.245688. PMID: 33054108; PMCID: PMC7716373.

17. Limaye AP, Bowden RA, Myerson D, Boeckh M. Cytomegalovirus disease occurring before engraftment in marrow transplant recipients. Clin Infect Dis. 1997 May;24(5):830-5. doi: 10.1093/clinids/24.5.830. PMID: 9142777.

18. Solano C, Giménez E, Albert E, Mateo EM, Gómez M, Goterris R, Pérez A, et al. Pre-engraftment cytomegalovirus DNAemia in allogeneic hematopoietic stem cell transplant recipients: incidence, risk factors, and clinical outcomes. Bone Marrow Transplant. 2019 Jan;54(1):90-98. doi: 10.1038/s41409-018-0251-0. Epub 2018 Jun 13. PMID: 29899574.

19. Martin I, Valsamakis A, Gladstone D, Jones R, Ambinder R, Avery RK. Cytomegalovirus in Adult Allogeneic Blood and Marrow Transplant Patients Before or Around the Period of Neutrophil Recovery: A Single-Center, Retrospective, Descriptive Study. Open Forum Infect Dis. 2020 Mar 9;7(3):ofaa081. doi: 10.1093/ofid/ofaa081. PMID: 32258204; PMCID: PMC7096132.

20. Goldsmith SR, Abid MB, Auletta JJ, Bashey A, Beitinjaneh A, Castillo P, et al. Post-Transplant Cyclophosphamide (PTCy) is Associated with Increased Cytomegalovirus Infection: A CIBMTR Analysis. Blood. 2021 Mar 3:blood.2020009362. doi: 10.1182/blood.2020009362. Epub ahead of print. PMID: 33657221.

21. Crawford LB, Tempel R, Streblow DN, Yurochko AD, Goodrum FD, Nelson JA, Caposio P. Human Cytomegalovirus Infection Suppresses $\mathrm{CD} 34^{+}$Progenitor Cell Engraftment in Humanized Mice. Microorganisms. 2020 Apr 6;8(4):525. doi: 10.3390/microorganisms8040525. PMID: 32268565; PMCID: PMC7232458.

22. Maciejewski JP, St Jeor SC. Human cytomegalovirus infection of human hematopoietic progenitor cells. Leuk Lymphoma. 1999 Mar;33(1-2):1-13. doi: 10.3109/10428199909093720. PMID: 10194116.

23. Cantoni N, Hirsch HH, Khanna N, Gerull S, Buser A, Bucher C, et al. Evidence for a bidirectional relationship between cytomegalovirus replication and acute graft-versus-host disease. Biol Blood Marrow Transplant. 2010 Sep;16(9):1309-14. doi: 10.1016/j.bbmt.2010.03.020. Epub 2010 Mar 29. PMID: 20353832.

24. Marty FM, Ljungman P, Chemaly RF, Maertens J, Dadwal SS, Duarte RF, et al. Prophylaxis for Cytomegalovirus in Hematopoietic-Cell Transplantation. N Engl J Med. 2017 Dec 21;377(25):2433- 
2444. doi: 10.1056/NEJMoa1706640. Epub 2017 Dec 6. PMID: 29211658.

25. Marty FM, Ljungman PT, Chemaly RF, Wan H, Teal VL, Butterton JR, et al. Outcomes of patients with detectable CMV DNA at randomization in the phase III trial of letermovir for the prevention of CMV infection in allogeneic hematopoietic cell transplantation. Am J Transplant. 2020 Jun;20(6):17031711. doi: 10.1111/ajt.15764. Epub 2020 Jan 18. PMID: 31883426.

Figures

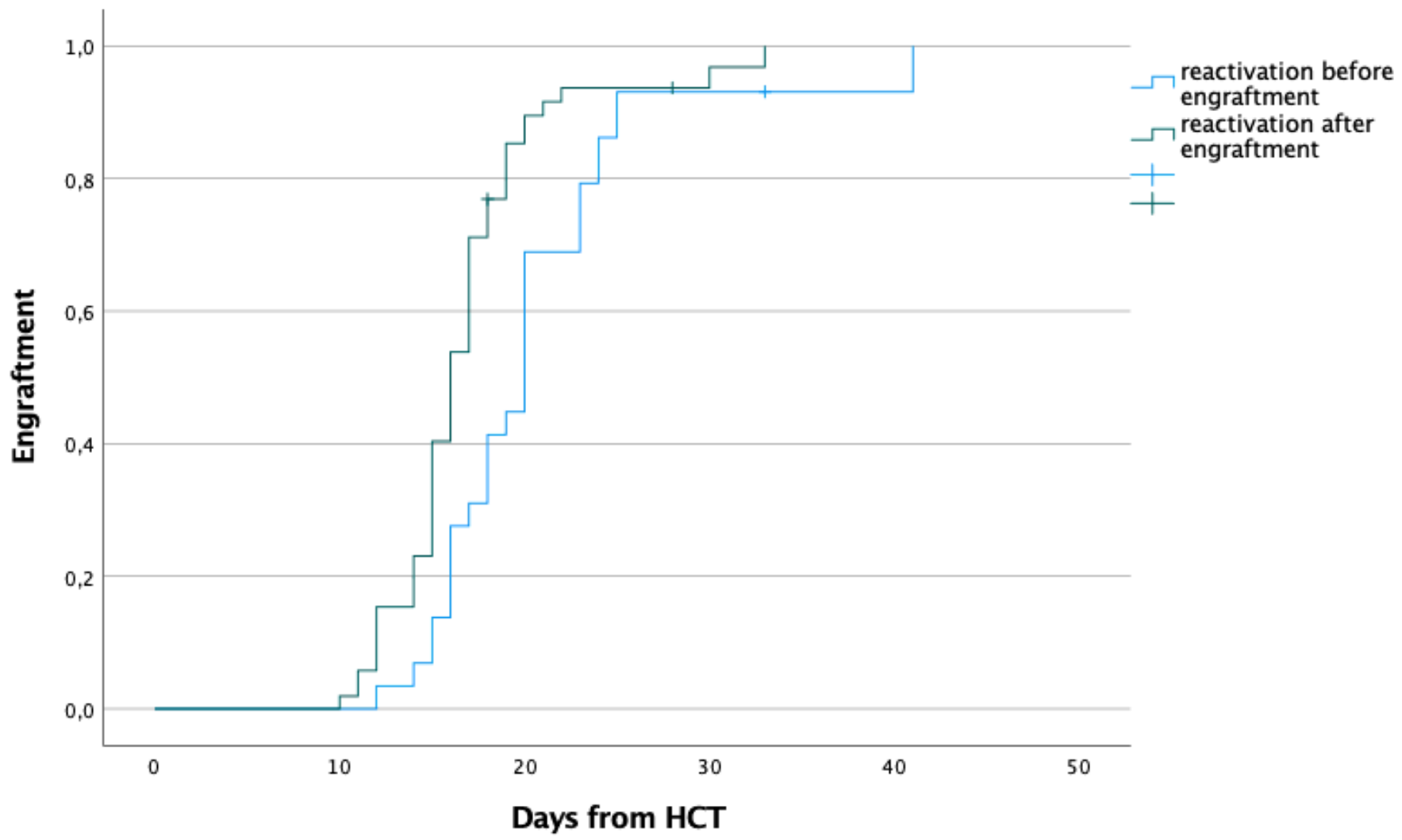

\section{Figure 1}

Time to engraftment probability among allogeneic HCT recipients stratified by pre-engraftment and postengraftment csCMVi 


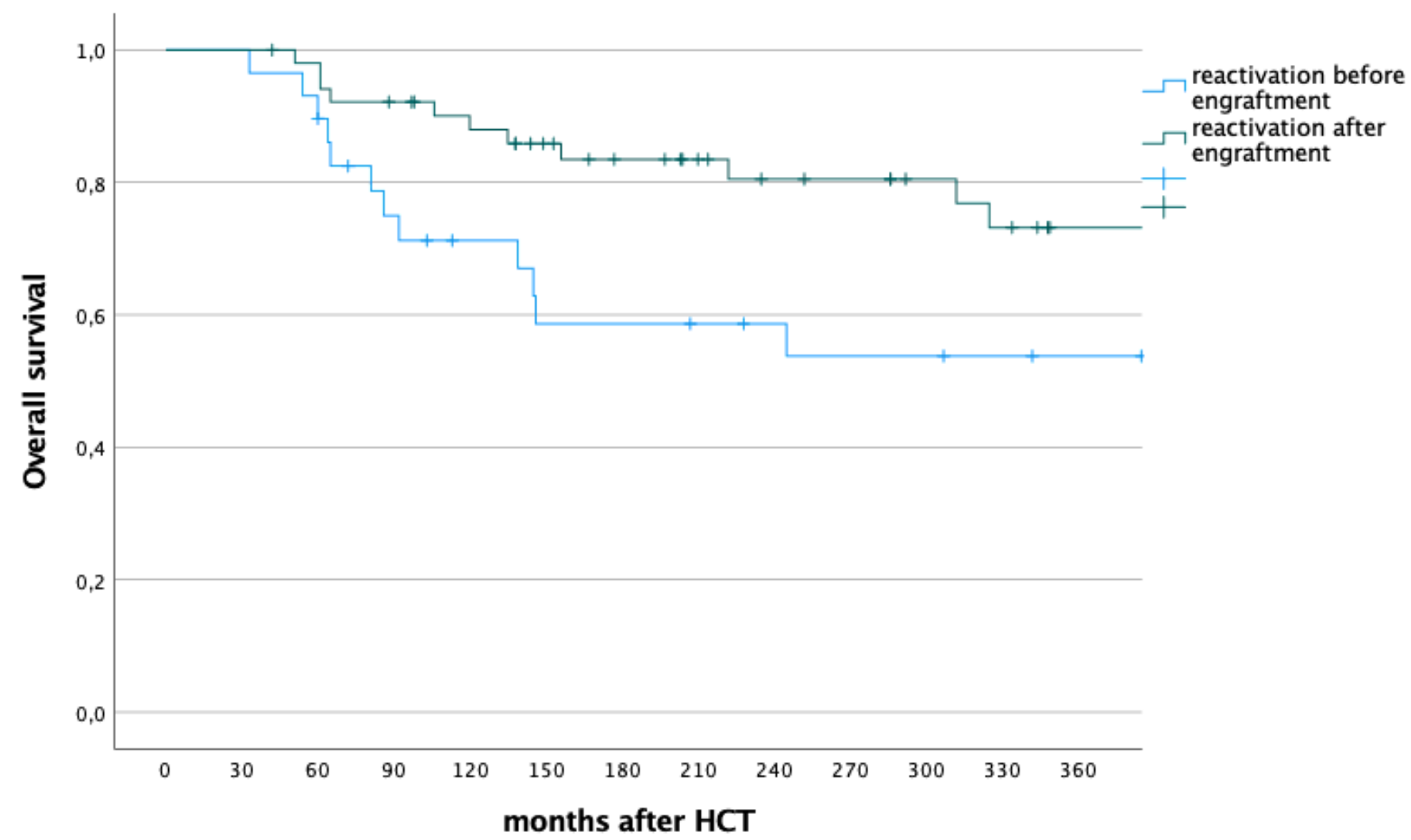

Figure 2

Overall survival probability among allogeneic HCT recipients stratified by pre-engraftment and postengraftment csCMVi 